\title{
Characterization of InGaAs and InGaAsN semiconductor saturable absorber mirrors for high-power mode-locked thin-disk lasers
}

\author{
F. Schättiger · D. Bauer · J. Demsar · T. Dekorsy • \\ J. Kleinbauer · D.H. Sutter • J. Puustinen • M. Guina
}

\begin{abstract}
We report a comparative study of carrier dynamics in semiconductor saturable absorber mirrors (SESAMs) containing InGaAs quantum wells and InGaAsN quantum wells (QWs). The static and dynamic reflectivity spectra were measured with a Fourier-transform-infraredspectrometer and a pump-probe setup, respectively. The influence of rapid thermal annealing (RTA) on carrier dynamics was studied. Due to the reduction of defect states by RTA we observed an increase of the static reflectivity and an increase of the electron-hole recombination time.

We demonstrate that nitrogen incorporation causes a decrease of the static reflectivity of the SESAMs, an increase of the modulation depth, and a reduction of the carriers' recombination time. We also investigated the mode-locking behavior of the SESAMs in an Yb:YAG thin-disk laser oscillator. The highest pulse energies directly obtained from a laser oscillator under stable operation conditions were achieved when using a SESAM with InGaAsN quantum wells.
\end{abstract}

F. Schättiger ( $₫)$ D. Bauer · J. Demsar · T. Dekorsy

Center for Applied Photonics and Department of Physics,

University Konstanz, Box M700, 78457 Konstanz, Germany

e-mail: farina.schaettiger@uni-konstanz.de

Fax: +49-7531-883072

D. Bauer $\cdot$ J. Kleinbauer $\cdot$ D.H. Sutter

TRUMPF Laser GmbH \& Co. KG, 78713 Schramberg, Germany

J. Puustinen · M. Guina

Tampere University of Technology, Korkeakoulunkatu 3,

33720 Tampere, Finland

J. Puustinen $\cdot$ M. Guina

RefleKron Ltd., Muotialankuja 5 C5, 33800 Tampere, Finland

\section{Introduction}

Semiconductor saturable absorber mirrors (SESAMs) are common elements for passively mode-locking lasers [1-3]. They contain a semiconductor Bragg mirror and on top a quantum well absorber which can be bleached. SESAMs allow for self-starting mode-locked lasers emitting ultrashort pulses in the subpicosecond regime with a compact and simple architecture. The mode-locking behavior of a laser depends on the modulation depth, the saturation fluence, and the recovery time of the SESAM [4]. The first two of these parameters describe the nonlinear reflectivity behavior of the SESAM [5, 6]. Since for stable and self-starting pulse shaping a fast recovery time is desirable [1], in the last decades different ways to reduce the recovery time were investigated such as quantum dot SESAMs [7], low temperature growth [8-10], or nitrogen $(\mathrm{N})$ incorporation into the quantum wells [11, 12].

The SESAMs investigated in this work were designed for a lasing wavelength of $1030 \mathrm{~nm}$. A suitable absorption material for this wavelength is indium gallium arsenide $\left(\operatorname{In}_{x} \mathrm{Ga}_{1-x} \mathrm{As}\right)$. Its band gap energy decreases with increasing indium content. With decreasing band gap energy the lattice constant increases and causes a lattice mismatch when grown on gallium arsenide (GaAs). This deviation of the lattice constant to that of GaAs can be compensated when nitrogen is added. For a certain In composition, alloying $\mathrm{N}$ to InGaAs leads also to a decrease of the band gap energy and an increase of the conduction band offset when increasing the nitrogen content [13]. This in turn leads to stronger carrier confinement, higher carrier density, and hence improved temperature behavior of the absorption/emission properties. Incorporation of nitrogen into the InGaAs lattice is usually accompanied by generation of non-radiative recombination centers which have detrimental effects on the emission effi- 
ciency but could be positively explored for ultrafast nonlinear optical applications. Since nitrogen incorporation causes defects which act as non-radiative recombination centers, one can control the electron-hole recombination time by adding a certain amount of nitrogen or by other growthrelated techniques $[11,14]$.

Another way of changing the characteristics of a SESAM is post-growth annealing. Photoluminescence (PL) measurements of semiconductor materials have shown that post-growth annealing causes (i) an improvement of the PL-efficiency [15-20], which implies a reduction of nonradiative point defects and stress; (ii) a reduction of the spectral linewidth $[15,16]$; (iii) a blue shift of the PL-peak $[18,19]$ mainly due to an increase of the band gap; and (iv) an interdiffusion of the atoms [21]. By these effects the response time as well as the absorption wavelength of the SESAM can be manipulated.

In this paper we report a comparative study of carrier dynamics and optical properties of SESAMs with $\mathrm{In}_{0.3} \mathrm{Ga}_{0.7} \mathrm{As}$ and $\mathrm{In}_{0.3} \mathrm{Ga}_{0.7} \mathrm{As}_{0.985} \mathrm{~N}_{0.015}$ quantum wells used for passive mode-locking of a diode-pumped Yb:YAG thin-disk laser oscillator generating subpicosecond pulses with pulse energies up to $30 \mu \mathrm{J}$ with a repetition rate of $3.5 \mathrm{MHz}$ [22]. These results were achieved with the laser operating in ambient atmosphere without signs of damage on the SESAM.

Different measurements have been performed in order to obtain the static reflectivity and the dynamic reflectivity changes of the SESAMs. The dynamic measurements were carried out at $1030 \mathrm{~nm}$, which is the operation wavelength of the $\mathrm{Yb}$ :YAG thin-disk oscillator. The most intriguing point we aim to investigate is the degree of change of optical properties, i.e. reflectivity, caused by thermal annealing. Such changes could also occur during laser operation due to aging of the SESAM and could be important for long-term laser operation of a SESAM-based laser, where stable static and dynamic properties are required.

\section{SESAM structures}

We tested two different kinds of SESAMs which were otherwise similar except for the type of QWs used. The two structures were comprised of a Bragg reflector with 27 pairs of GaAs/AlAs $\lambda / 4$ layers and a GaAs absorption layer incorporating the $\mathrm{QW}$ absorbing regions. The absorbing region for SESAMs R1 contained five $\mathrm{In}_{0.3} \mathrm{Ga}_{0.7}$ As QWs with a maximum photoluminescence (PL) emission at about $1080 \mathrm{~nm}$. The SESAM termed R2 had the same design as the type $\mathrm{R} 1$, except for the QWs which were based on a dilute nitride $\left(\mathrm{In}_{0.3} \mathrm{Ga}_{0.7} \mathrm{As}_{0.985} \mathrm{~N}_{0.015}\right)$ material system. The indium mole fraction for the two types of quantum wells was similar (of about $30 \%$ ). Incorporation of $\mathrm{N}$ in the range of $1.2-1.5 \%$ led to a strong red shift of the PL emission, which exhibited a maximum at about $>1150 \mathrm{~nm}$.
The quantum wells of the InGaAs structure have been grown at a temperature of $520^{\circ} \mathrm{C}$. This temperature ensures a good optical quality. The quantum wells of the InGaAsN structure have been grown at lower temperature, i.e. $460^{\circ} \mathrm{C}$, which is optimal in terms of optical quality. The distributed Bragg reflector (DBR) and the GaAs layers surrounding the $\mathrm{QWs}$ have been grown at $680^{\circ} \mathrm{C}$. After growth different asgrown samples were annealed at $450^{\circ} \mathrm{C}, 650^{\circ} \mathrm{C}$, and $700^{\circ} \mathrm{C}$ for $100 \mathrm{~s}$.

\section{Experimental setup}

The static reflectivity was measured with a Fourier-transform-infrared-reflectivity-spectrometer (Bruker Vertex 70). For reflectivity calibration a gold mirror was used.

The dynamic reflectivity changes were measured with a degenerate pump-probe setup at the wavelength of $1030 \mathrm{~nm}$. The samples R1 were measured with a setup using a tunable optical parametric amplifier (OPA) pumped by a $250 \mathrm{kHz}$ regenerative Ti:Sapphire amplifier as laser source, delivering $70 \mathrm{fs}$ pulses with a spectral width of $25 \mathrm{~nm}$. With this setup a time-delay window of 15 ps was covered using a mechanical delay scanner (APE ScanDelay $15 \mathrm{ps}$ ).

The measurements of the SESAMs of type R2 were done using an Yb-doped fiber laser emitting 320 fs laser pulses with a wavelength of $1030 \mathrm{~nm}$ and a spectral width of $7 \mathrm{~nm}$. The scan range of the time delay between pump and probe beam was enlarged to $50 \mathrm{ps}$ (APE ScanDelay $50 \mathrm{ps}$ ).

Fluences from $5 \mu \mathrm{J} / \mathrm{cm}^{2}$ to $450 \mu \mathrm{J} / \mathrm{cm}^{2}$ were covered. The beam spots were carefully determined with pinholes of different sizes. The pump beam had a pulse energy density of more than $20 \mu \mathrm{J} / \mathrm{cm}^{2}$, while the probe beam fluences were kept smaller than $3 \mu \mathrm{J} / \mathrm{cm}^{2}$. The pump and probe pulses were cross-polarized in order to reduce the coherent artifact [23].

\section{Results}

The two different investigated SESAMs show quite different changes of the optical properties with annealing. The properties of the samples $\mathrm{R} 1$ with $\mathrm{In}_{0.3} \mathrm{Ga}_{0.7}$ As quantum wells were almost unchanged with annealing, whereas samples of type R2 with $\operatorname{In}_{0.3} \mathrm{Ga}_{0.7} \mathrm{As}_{0.985} \mathrm{~N}_{0.015}$ quantum wells change significantly upon annealing.

\subsection{Static reflectivity}

In Fig. 1 the static reflectivity spectra of both the InGaAs and the InGaAsN absorber based SESAMs are shown for different post-growth annealing temperatures. The as-grown sample of R1 was not available for the static reflectivity measurements. Since the dynamic reflectivity of the unannealed 

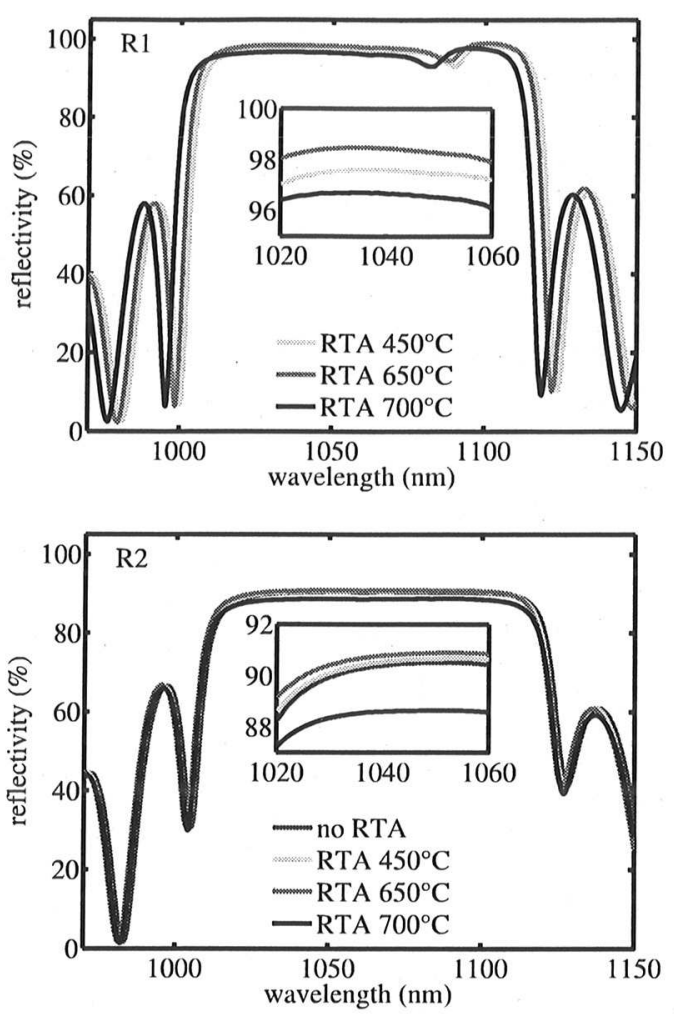

Fig. 1 Static reflectivity spectra of both investigated SESAMs at different annealing temperatures. Top: SESAM R1 with $\operatorname{In}_{0.3} \mathrm{Ga}_{0.7} \mathrm{As}$ QWs. Bottom: SESAM R2 with $\operatorname{In}_{0.3} \mathrm{Ga}_{0.7} \mathrm{As}_{0.985} \mathrm{~N}_{0.015}$ QWs. Insets: Zoom into the stop band regions

R1 does not differ much from the SESAM R1 annealed at $450^{\circ} \mathrm{C}$ (see Sect. 4.2), the static reflectivity spectra of both are expected to be similar as well.

Figure 1 shows that the static reflectivity of SESAMs R1 and $\mathrm{R} 2$ has its maximum in the wavelength range between 1020 and $1100 \mathrm{~nm}$. The weak minima in the reflectivity of $\mathrm{R} 1$ at a wavelength of $1090 \mathrm{~nm}$ are caused by the excitonic resonance of the quantum well absorber. The spectrum of the SESAMs of type R2 based on an InGaAsN absorbers does not show any visible dip in the high reflectivity part of the spectrum. According to the design of R2 the excitonic resonance of the quantum well is expected at around $1190 \mathrm{~nm}$, i.e. outside the high reflectivity part of the Bragg reflector, as confirmed in photoluminescence (PL) measurements.

For all the samples the excitonic resonance is more than a longitudinal optical (LO) phonon energy of InGaAs(N) below the excitation wavelength. Therefore the relaxation within the conduction band of the highly excited electrons is possible via a LO phonon emission.

In general the static reflectivity of all SESAMs of type $\mathrm{R} 2$ is less compared to that of the SESAMs R1. This observation corresponds to the fact that adding $\mathrm{N}$ into InGaAs increases the density of states near the band gap [13]. Therefore the absorption of InGaAsN QWs is higher resulting in a lower reflectivity. The higher density of states of InGaAsN can also account for the changes of the dynamic reflectivity, as will be discussed in Sect. 4.2.

Changes in the static reflectivity due to thermal annealing can clearly be seen in the insets of Fig. 1. For R2 annealing at $450^{\circ} \mathrm{C}$ does not result in a change of reflectivity compared with the as-grown sample. Independently of the quantum well material, the reflectivity increases with increasing the RTA temperature up to $650^{\circ} \mathrm{C}$. At RTA temperature of $700^{\circ} \mathrm{C}$ the reflectivity drops down to a value lower than that of the as-grown sample. This behavior shows that the process of annealing decreases the amount of defect states in the structure and consequently causes an increase of the reflectivity, as long as the RTA temperature is not too high. One reason for the decrease of reflectivity for RTA at $700^{\circ} \mathrm{C}$ might be the degradation of the AlAs/GaAs interfaces in the Bragg mirror, which could cause the difference of the effective refractive indexes to become smaller and thus result in a reflectivity decrease. However, this shift is not expected to influence the dynamic reflectivity change at $1030 \mathrm{~nm}$.

In the static reflectivity spectra of the SESAMs R1 in Fig. 1 a slight blue shift of the absorption minimum and the distributed Bragg reflector (DBR) with increasing RTA temperature are visible. A reason for the DBR wavelength shift might be related to flux non-uniformity of molecular beam epitaxy that leads to variation of parameters across the wafer. We also cannot fully exclude degradation of the surface quality due to As out-diffusion from the surface although all samples were placed in a proximity of GaAs wafer during the annealing process [20].

\subsection{Time-dependent reflectivity}

The dynamic properties of SESAMs are essential parameters for the lasing behavior. In Figs. 2 and 4 the transient reflectivity of the two SESAMs with different absorber material and annealing temperatures are shown for different pump fluences.

A typical transient of a SESAM looks as follows. During photoexcitation (zero-time delay) the reflectivity increases within the pump pulse duration up to a high value, because carriers are excited into high states within the conduction band and the absorbers are bleached. Within 60-100 fs the carriers thermalize and the reflectivity drops down a little bit. The fast time constant in the transients originates from this thermalization process. The slow time constant of the decay in the picosecond regime is attributed to electron recombination with holes in the valence band and trapping in point defects [24]. The instantaneous reflectivity change at zero-time delay increases with increasing pump fluences, as it can be seen in the nonlinear reflectivity curves of the SESAMs shown in Figs. 3 and 5.

For high pump fluences an additional effect appears: due to two-photon absorption (TPA) or free carrier absorption 

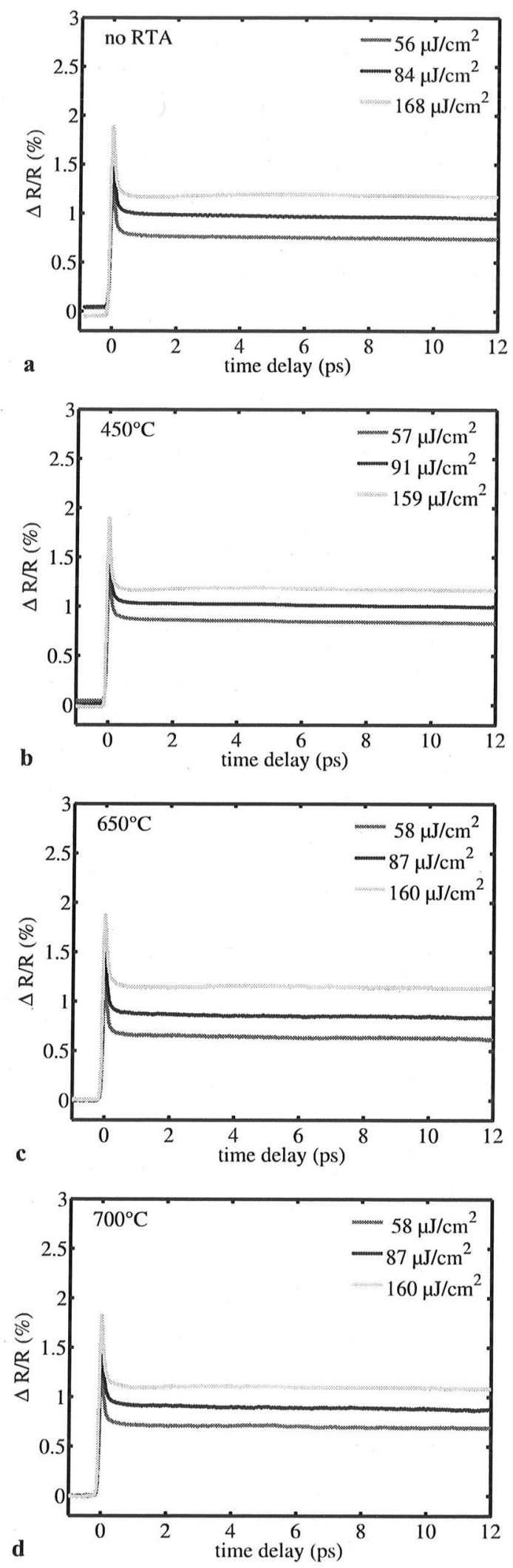

Fig. 2 Transient reflectivity of the $\operatorname{In}_{0.3} \mathrm{Ga}_{0.7} \mathrm{As}$ based absorber samples $\mathrm{R} 1$ at different annealing temperatures

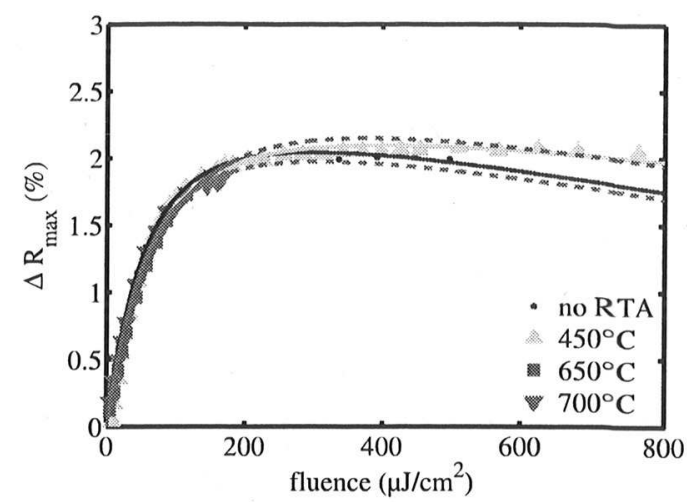

Fig. 3 Nonlinear reflectivity of all SESAMs of type R1. The markers indicate the measured data points, the lines are fits. Since for the measurements of the samples annealed at $650^{\circ} \mathrm{C}$ and $700^{\circ} \mathrm{C}$ only fluences below $180 \mu \mathrm{J} / \mathrm{cm}^{2}$ were available, the fits at higher fluences are less reliable

(FCA) the carriers are excited in even higher states within the conduction band, hence the absorber bleaching contribution is delayed [25-27]. In transient reflectivity these nonlinear effects cause (i) a decreased instantaneous reflectivity change, and (ii) a second rise of the reflectivity after the excitation. The second rise appears with a time constant of high-energy carriers relaxing from the $\mathrm{L}$ - and $\mathrm{X}$-valley of the conduction band. In the nonlinear reflectivity spectra the roll-over, i.e., the decrease of the initial reflectivity change at higher fluences is attributed to these nonlinear effects $[5,28]$.

The transient reflectivity of all samples of SESAM R1 with InGaAs QWs is shown in Fig. 2. As described above, a bi-exponential decay is existent for low excitation fluences; for higher fluences a slight second rise appears after approximately $4 \mathrm{ps}$ in the transient reflectivity of the unannealed and the RTA $-450^{\circ} \mathrm{C}$ sample. As the static reflectivity spectra of the time and fluence dependent behavior of the differently annealed SESAMs R1 show only small changes: the absolute amplitude and the shape of the transients change only barely with different annealing temperatures. Another evidence that the SESAMs R1 with InGaAs QWs do not change much with annealing are the unchanged relaxation times. Fitting the data with a double exponential decay results in a fast time constant of $<100$ fs and a slow time constant of a few hundred picoseconds, independent of the annealing temperature. This suggests almost no changes in the electronic relaxation dynamics.

Figure 3 shows the nonlinear reflectivity curves of the SESAMs R1. The experimental data points were fitted by the function

$$
\begin{aligned}
f(x)= & \frac{100 \ln \left[1+R_{\operatorname{lin}} / 100 \cdot\left(\exp \left(x / F_{\text {sat }}\right)-1\right)\right]}{x / F_{\text {sat }}} \\
& \cdot \exp \left(-x / F_{2}\right)-R_{\text {lin }},
\end{aligned}
$$


Table 1 Fit parameter and time constants of all SESAMs R1 with In $_{0.3} \mathrm{Ga}_{0.7} \mathrm{As}$ QWs. The fast and slow time constants are extracted from the transients obtained at fluences in the range of $90 \mu \mathrm{J} / \mathrm{cm}^{2}$ (see Fig. 2). For the SESAMs annealed at $650^{\circ} \mathrm{C}$ and $700^{\circ} \mathrm{C}$ we calculated the fit parameter for fixed $F_{2}=110 \mathrm{~mJ} / \mathrm{cm}^{2}$

\begin{tabular}{llllll}
\hline SESAM R1 & $\begin{array}{l}\Delta R \\
(\%)\end{array}$ & $\begin{array}{l}F_{\text {sat }} \\
\left(\mu \mathrm{J} / \mathrm{cm}^{2}\right)\end{array}$ & $\begin{array}{l}F_{2} \\
\left(\mathrm{~mJ} / \mathrm{cm}^{2}\right)\end{array}$ & $\begin{array}{l}\tau_{\text {fast }} \\
(\mathrm{ps})\end{array}$ & $\begin{array}{l}\tau_{\text {slow }} \\
(\mathrm{ps})\end{array}$ \\
\hline no RTA & 2.0 & 32 & 105 & 0.08 & $>200$ \\
$450^{\circ} \mathrm{C}$ & 2.1 & 36 & 170 & 0.07 & $>200$ \\
$650^{\circ} \mathrm{C}$ & 2.1 & 45 & 110 (fixed) & 0.09 & $>200$ \\
$700^{\circ} \mathrm{C}$ & 2.0 & 34 & 110 (fixed) & 0.09 & $>200$ \\
\hline
\end{tabular}

with $R_{\text {lin }}$ being the linear reflectivity without any optical excitation, $F_{\text {sat }}$ the saturation fluence, and $F_{2}$ the parameter for induced absorption [5]. Since for the measurements of the SESAMs R 1 annealed at $650^{\circ} \mathrm{C}$ and $700^{\circ} \mathrm{C}$ no fluence values above $180 \mu \mathrm{J} / \mathrm{cm}^{2}$ could be achieved, we used a fixed value for $F_{2}$ in the fitting function. In correspondence to the other two samples we consider $F_{2}=110 \mathrm{~mJ} / \mathrm{cm}^{2}$ as reasonable.

From the nonlinear reflectivity curve the modulation depth of a SESAM can be extracted: it corresponds to the highest change in reflectivity. The modulation depth of any SESAM of type R1 was approximately $2.0 \%$. Since the static reflectivity of R1 was approximately $98 \%$, that would mean that the non-saturable losses of SESAMs R1 are very small, i.e. within the error of determining $100 \%$ reflectivity. All parameters of the SESAMs of type R1 are listed in Table 1.

Figure 4 shows the transients of all samples of SESAM R2 containing $\operatorname{In}_{0.3} \mathrm{Ga}_{0.7} \mathrm{As}_{0.985} \mathrm{~N}_{0.015} \mathrm{QWs}$. The transients show an increase of the instantaneous reflectivity change with increasing pump fluences. Compared to the transients in Fig. 2, the fast time constant of the reflectivity decay in Fig. 4 is nearly invisible. For fluences below $400 \mu \mathrm{J} / \mathrm{cm}^{2}$ the fast time constant cannot be resolved due to a pump- and probe-pulse length of $320 \mathrm{fs}$. In the transients corresponding to the fluence of $442 \mu \mathrm{J} / \mathrm{cm}^{2}$, the fast time constant is almost completely dominated by the second rise of the reflectivity caused by FCA and TPA.

In contrast to the behavior of the SESAMs R1, the transients of the SESAMs R2 with InGaAsN QWs change significantly with annealing temperatures. With increasing RTA temperature the recombination time rises, visible in the slow decay of the transients. This can be attributed to the annealing of point defects incorporated by the low growth temperature of the quantum wells. For example, at a fluence of $88 \mu \mathrm{J} / \mathrm{cm}^{2}$ the slow time constant of the as-grown sample is ca. $20 \mathrm{ps}$, whereas it is ca. $130 \mathrm{ps}$ for the sample annealed at $700^{\circ} \mathrm{C}$. Another effect of increasing RTA temperature is a slight enhancement of the second rise of the reflectivity due to TPA and FCA. For RTA temperatures above $450^{\circ} \mathrm{C}$ the
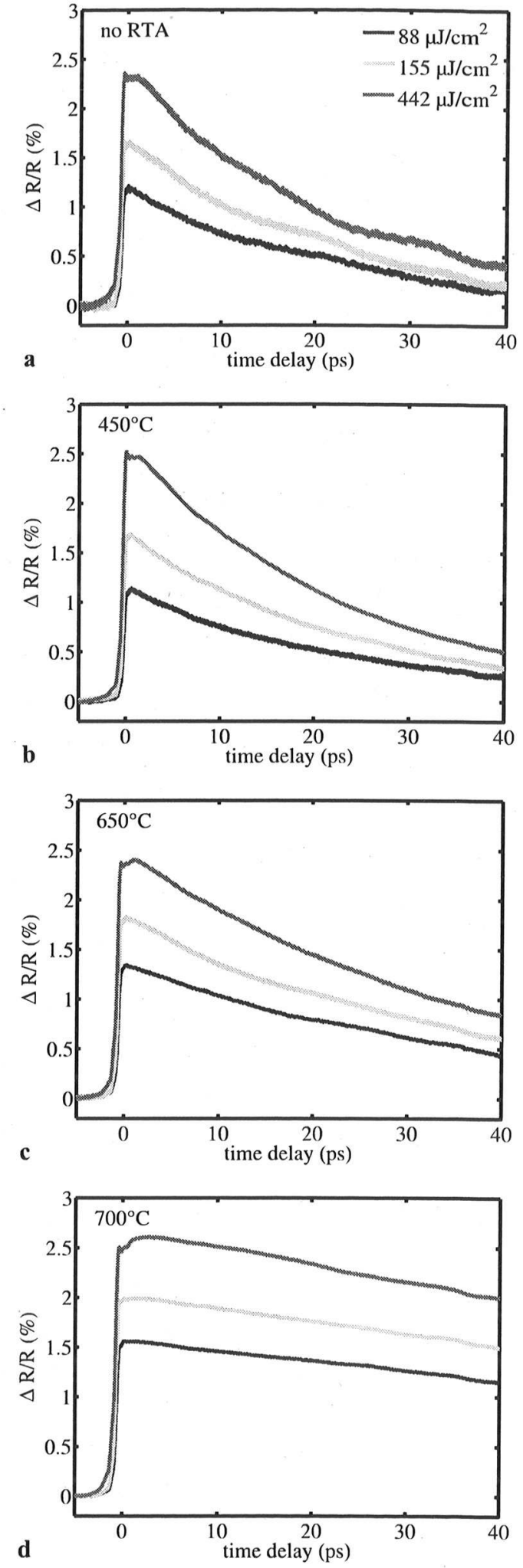

Fig. 4 Transient reflectivity of the $\mathrm{In}_{0.3} \mathrm{Ga}_{0.7} \mathrm{As}_{0.985} \mathrm{~N}_{0.015}$ based absorber samples R2 at different annealing temperatures and pump fluences. The legend in (a) is valid for (b)-(d) as well 


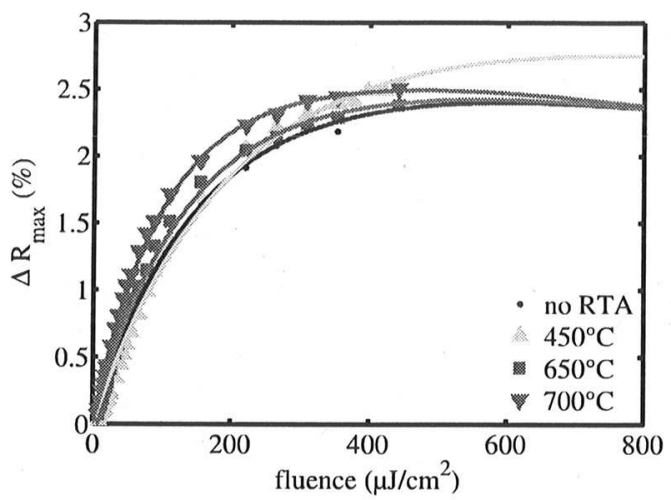

Fig. 5 Nonlinear reflectivity of all SESAMs of type R2. The markers indicate the measured data points, the lines are fits

Table 2 Fit parameter and time constants of all SESAMs R2 with $\mathrm{In}_{0.3} \mathrm{Ga}_{0.7} \mathrm{As}_{0.985} \mathrm{~N}_{0.015}$ QWs. The fast time constant could not be extracted from the transients. The slow time constants belong to the transients at a fluence of $88 \mu \mathrm{J} / \mathrm{cm}^{2}$ (see Fig. 4). We calculated the fit parameters again for $F_{2}$ fixed at $110 \mathrm{~mJ} / \mathrm{cm}^{2}$

\begin{tabular}{lllll}
\hline SESAM R2 & $\begin{array}{l}\Delta R \\
(\%)\end{array}$ & $\begin{array}{l}F_{\text {sat }} \\
\left(\mu \mathrm{J} / \mathrm{cm}^{2}\right)\end{array}$ & $\begin{array}{l}F_{2} \\
\left(\mathrm{~mJ} / \mathrm{cm}^{2}\right)\end{array}$ & $\begin{array}{l}\tau_{\text {slow }} \\
(\mathrm{ps})\end{array}$ \\
\hline no RTA & 2.4 & 94 & 110 (fixed) & 21 \\
$450^{\circ} \mathrm{C}$ & 2.7 & 125 & 110 (fixed) & 27 \\
$650^{\circ} \mathrm{C}$ & 2.4 & 82 & 110 (fixed) & 37 \\
$700^{\circ} \mathrm{C}$ & 2.5 & 61 & 110 (fixed) & 132 \\
\hline
\end{tabular}

reflectivity change of the second rise is even higher than the instantaneous reflectivity at zero-time delay.

As it can be seen in Fig. 4, the signal amplitude for a given fluence value is independent of the RTA temperature of the SESAM. For this reason the nonlinear reflectivity curves of the R2 samples are quite homogeneous, as it is shown in Fig. 5. The effective modulation depths of all SESAMs of type R2 are ca. $2.5 \%$. Due to the static reflectivity of R2 in the range of $90 \%$ and less, we conclude that the InGaAsN-SESAMs have more than $6 \%$ of non-saturable losses. The faster increase of the nonlinear reflectivity of the SESAM R2 annealed at $700^{\circ} \mathrm{C}$ corresponds to the lower saturation fluence. Even at a pump fluence of $400 \mu \mathrm{J} / \mathrm{cm}^{2}$ we were still not able to see any roll-over due to TPA or FCA in the nonlinear reflectivity. Therefore we fitted the data of these samples with a fixed $F_{2}=110 \mathrm{~mJ} / \mathrm{cm}^{2}$ in correspondence to the $F 2$ parameters of the samples R1. All parameters of the samples $\mathrm{R} 2$ are listed in Table 2.

Comparing the dynamic reflectivity of the SESAMs of type R1 and R2, four main differences are apparent. (i) The electron-hole recombination time of the samples $\mathrm{R} 1$ with a few hundred picoseconds is much higher than the recombination time of the SESAMs R2 with some tens of picoseconds. Due to nitrogen incorporation, defect states are in- corporated, which capture the excited electrons and accelerate the electron-hole recombination. (ii) The recombination time of the SESAMs R1 is hardly affected by thermal annealing, whereas the recombination time of the SESAMs $\mathrm{R} 2$ containing InGaAsN QWs rises by more than five times when annealed at $700^{\circ} \mathrm{C}$. The reason for the strong influence of RTA on the recombination time of the InGaAsN samples is attributed to the nitrogen. Albrecht et al. found out that RTA broadens the nitrogen concentration profile in InGaAsN quantum wells. Therefore the $\mathrm{N}$ concentration in the quantum well decreases and in conclusion the amount of recombination centers decreases. A thickness increase of the indium concentration profile due to thermal annealing could not be observed [21]. This may explain why the recombination time of the SESAMs R1 with InGaAs QWs does not change significantly with annealing. (iii) The modulation depths of the SESAMs R2, which are $2.5 \%$, are a little bit higher than those of the samples R1 with $2.0 \%$. The explanation for this lies in an increased absorption in the InGaAsN QWs compared to standard InGaAs QWs due to $\mathrm{N}$ diffusion from the QW region, and hence a thicker absorption region. (iv) The slower increase of the nonlinear reflectivity curves at low fluences of samples of type R2 compared to the curves of SESAMs R1 is reflected in higher saturation fluences. This effect and the difference in the static reflectivity can be explained by the density of states, which is higher in InGaAsN QWs than in InGaAs QWs [13].

\section{Mode-locking behavior}

The SESAM dynamics determine the mode-locking behavior of a laser. The problem, which has to be avoided, is Q-switching. The tendency to Q-switching instabilities of a mode-locked laser is affected by the saturation fluence and the modulation depth of its SESAM. A modified form of the criterion against $\mathrm{Q}$-switching, which was originally given by Hoenninger et al., is:

$E_{P}>\frac{\Delta R}{S} E_{\mathrm{sat}, L}, \quad$ with $S=\frac{E_{P}}{E_{\mathrm{sat}, A}}$

where $E_{P}$ is the energy of the intra-cavity pulse, $E_{\mathrm{sat}, L}$ is the energy of the gain, $\Delta R$ is the modulation depth, $S$ is the saturation parameter defined by the laser design, and $E_{\mathrm{sat}, A}$ is the absorber saturation energy [4].

This formula shows that with increasing the modulation depth and the saturation fluence, Q-switching is more likely. For that reason SESAMs of type R1 should give rise to more stable laser operation than SESAMs of type R2. This theoretical prediction could be verified in the thin-disk laser oscillator, where the tendency for Q-switching was higher when R2 was used for mode-locking. 
In general, both types of SESAMs (R1 with InGaAs and R2 with InGaAsN quantum wells) were used to mode-lock an Yb:YAG thin-disk laser with an active multipass cell [22, 29-31]. Mode-locking this type of laser requires a relatively high modulation depth, a high saturation fluence, negligible nonlinear roll-over and low non-saturable losses. Especially for the generation of subpicosecond-pulse SESAMs with modulation depths exceeding $1 \%$ were necessary. With a SESAM of type R2 with InGaAsN QWs and $\Delta R=2.5 \%$, laser pulses with a pulse energy of $30.7 \mu \mathrm{J}$ and a pulse length of $1040 \mathrm{fs}$ were achieved. To our knowledge, those are the highest pulse energies ever achieved directly out of a modelocked solid-state laser [22]. SESAM R1 with InGaAs QWs and $\Delta R=2.0 \%$ produced laser pulses of $953 \mathrm{fs}$ at pulse energies of $18.6 \mu \mathrm{J}$ [29]. Both SESAMs showed few defective areas after long-term use in the laser oscillator under lab conditions including laser adjustment, operation under Q-switching conditions and different beam spot sizes on the SESAMs.

\section{Summary}

In conclusion, the static as well as the dynamic reflectivity of SESAMs containing InGaAsN QWs differs from SESAMs with InGaAs QWs with respect to the reflectivity in general and with respect to the influence of thermal annealing and their optical properties in particular.

We measured the static and dynamic reflectivity of two types of SESAMs, R1 based on $\mathrm{In}_{0.3} \mathrm{Ga}_{0.7} \mathrm{As}$ as absorber material and $\mathrm{R} 2$ containing $\mathrm{In}_{0.3} \mathrm{Ga}_{0.7} \mathrm{As}_{0.985} \mathrm{~N}_{0.15}$ quantum wells as absorber. For each type we measured four postgrowth samples annealed at different temperatures: no annealing at all, annealed at $450^{\circ} \mathrm{C}, 650^{\circ} \mathrm{C}$, and $700^{\circ} \mathrm{C}$. The decrease of defect states due to thermal annealing is visible in an increase of the static reflectivity of the different SESAM samples until the annealing temperature of $700^{\circ} \mathrm{C}$. For the samples annealed at $700^{\circ} \mathrm{C}$-independently of the absorber material - the static reflectivity dropped down to a minimum value which might be attributed to the degradation of GaAs/AlAs layers in the DBR or surface degradation due to As desorption. The static reflectivity spectra of SESAMs of type R2 with InGaAsN QWs are in general lower than those of the SESAMs of type R1. The reason for this effect is attributed to the higher density of states in InGaAsN than in InGaAs.

The dynamic reflectivity of SESAMs R1 and R2 differs quite strongly in terms of the electron-hole recombination time, the modulation depth, the saturation fluence, and the influence of RTA. The measurements show that adding little amounts of nitrogen to InGaAs absorbers reduces the electron-hole recombination time. This is caused by the nitrogen atoms which act as recombination centers. An in- crease of the modulation depth for InGaAsN SESAMs compared to InGaAs SESAMs could be seen. Since the density of states in InGaAsN is higher than in InGaAs, the saturation fluences of the SESAMs R2 are higher than those of the SESAMs R1.

Whereas the dynamic reflectivity of the SESAMs R1 is hardly affected by thermal annealing processes, the electron-hole recombination time of the R2 SESAMs increases with increasing RTA temperature. The reason for that effect could be a decrease of the nitrogen atoms as recombination centers. Such a RTA-induced diffusion of the nitrogen atoms out of the quantum wells was observed by Albrecht et al. [21].

In particular, the differences in modulation depths, saturation fluences and the electron-hole recombination times might affect the use of the different types of SESAMs for mode-locking lasers: since for the self-starting of subpicosecond soliton-mode-locked pulses higher modulation depths are desirable [30], SESAMs of type R2 are more suitable as they provide a higher mode-locking driving force. The trade-offs of SESAMs R2 are the higher tendencies for Q-switching instabilities due to higher modulation depths and higher saturation fluences [4].

Acknowledgements We thank $\mathrm{M}$. Beyer and H. Schäfer for access to the optical parametric amplifier and JTEO for the use of the Ybdoped fiber laser. Financial support of the Center for Applied Photonics (CAP) is gratefully acknowledged.

\section{References}

1. U. Keller, K. Weingarten, F. Kärtner, D. Kopf, B. Braun, I. Jung, R. Fluck, C. Hönninger, N. Matuschek, J.A. der Au, IEEE J. Sel. Top. Quantum Electron. 2, 435 (1996)

2. F. Kärtner, I. Jung, U. Keller, IEEE J. Sel. Top. Quantum Electron. 2, 540 (1996)

3. F. Kärtner, J. Aus der Au, U. Keller, IEEE J. Sel. Top. Quantum Electron. 4, 159 (1998)

4. C. Hönninger, R. Paschotta, F. Morier-Genoud, M. Moser, U. Keller, J. Opt. Soc. Am. B, Opt. Phys. 16, 46 (1999)

5. M. Haiml, R. Grange, U. Keller, Appl. Phys. B, Lasers Opt. 79, 331 (2004)

6. D. Maas, B. Rudin, A. Bellancourt, D. Iwaniuk, S. Marchese, T. Südmeyer, U. Keller, Opt. Express 16, 7571 (2008)

7. M.P. Lumb, E. Clarke, E. Harbord, P. Spencer, R. Murray, F. Masia, P. Borri, W. Langbein, C.G. Leburn, C. Jappy, N.K. Metzger, C.T.A. Brown, W. Sibbett, Appl. Phys. Lett. 95, 041101 (2009). doi:10.1063/1.3186081. URL http://link.aip.org/link/ ?APL/95/041101/1

8. U. Siegner, R. Fluck, G. Zhang, U. Keller, Appl. Phys. Lett. 69, 2566 (1996)

9. M. Haiml, U. Siegner, F. Morier-Genoud, U. Keller, M. Luysberg, R. Lutz, P. Specht, E. Weber, Appl. Phys. Lett. 74, 3134 (1999)

10. A. Jasik, J. Muszalski, K. Pierściński, M. Bugajski, V. Talalaev, M. Kosmala, J. Appl. Phys. 106, 053101 (2009)

11. M. Guina, P. Tuomisto, O. Okhotnikov, S. Marcinkevicius, K. Mizohata, J. Keinonen, Proc. SPIE 6451 (2007). doi:10.1117/12. 725397 
12. C. Hsu, J. Lin, Y. Chen, Y. Lin, H. Kuo, S. Wang, W. Hsieh, N Tansu, L. Mawst, J. Phys. D, Lond. 41, 85107 (2008)

13. W. Walukiewicz, eScholarship Repository (University of California, Los Angeles, 2002)

14. T. Hakulinen, R. Herda, O. Okhotnikov, Appl. Opt. 47, 1235 (2008). URL http://ao.osa.org/abstract.cfm?URI=ao-47-9-1235

15. J.S. Wang, H.H. Lin, J. Vac. Sci. Technol., B Microelectron. Process. Phenom. 17(5), 1997 (1999). doi:10.1116/1.590860. URL http://link.aip.org/link/?JVB/17/1997/1

16. H.P. Xin, K.L. Kavanagh, C.W. Tu, J. Cryst. Growth 208, 145 (2000). doi:10.1016/S0022-0248(99)00415-7

17. W. Li, M. Pessa, T. Ahlgren, J. Decker, Appl. Phys. Lett. 79, 1094 (2001). doi:10.1063/1.1396316. URL http://link.aip.org/ link/?APL/79/1094/1

18. H.D. Sun, R. Macaluso, S. Calvez, G.J. Valentine, D. Burns, M.D. Dawson, K. Gundogdu, K.C. Hall, T.F. Boggess, T. Jouhti, M. Pessa, J. Appl. Phys. 96, 1418 (2004). doi:10.1063/1.1767612. URL http://link.aip.org/link/?JAP/96/1418/1

19. V. Liverini, A. Rutz, U. Keller, S. Schön, J. Appl. Phys. 99, 113103 (2006)

20. J. Pakarinen, C.S. Peng, J. Puustinen, P. Laukkanen, V.M. Korpijärvi, A. Tukiainen, M. Pessa, Appl. Phys. Lett. 92, 232105 (2008). doi:10.1063/1.2943157. URL http://link.aip.org/link/ ?APL/92/232105/1

21. M. Albrecht, V. Grillo, T. Remmele, H.P. Strunk, A.Y. Egorov, G. Dumitras, H. Riechert, A. Kaschner, R. Heitz, A. Hoff- mann, Appl. Phys. Lett. 81, 2719 (2002). doi:10.1063/1.1509122. URL http://link.aip.org/link/?APL/81/2719/1

22. D. Bauer, F. Schättiger, J. Kleinbauer, D. Sutter, A. Killi, T. Dekorsy, in ASSP (2011), p. ATuC2

23. M. Lebedev, O. Misochko, T. Dekorsy, N. Georgiev, J. Exp. Theor Phys. 100(2), 272 (2005)

24. U. Keller, Nature 424, 831 (2003). URL http://dx.doi.org/10 1038/nature01938

25. E. Thoen, E. Koontz, M. Joschko, P. Langlois, T. Schibli, F. Kärtner, E. Ippen, L. Kolodziejski, Appl. Phys. Lett. 74, 3927 (1999)

26. P. Langlois, M. Joschko, E. Thoen, E. Koontz, F. Kärtner, E. Ippen, L. Kolodziejski, Appl. Phys. Lett. 75, 3841 (1999)

27. M. Joschko, P. Langlois, E. Thoen, E. Koontz, E. Ippen, L. Kolodziejski, Appl. Phys. Lett. 76, 1383 (2000)

28. R. Grange, M. Haiml, R. Paschotta, G. Sphler, L. Krainer, M. Golling, O. Ostinelli, U. Keller, Appl. Phys. B, Lasers Opt. 80, 151 (2005)

29. J. Neuhaus, J. Kleinbauer, A. Killi, S. Weiler, D. Sutter, T. Dekorsy, Opt. Lett. 33, 726 (2008). URL http://ol.osa.org/abstract. cfm?URI=ol-33-7-726

30. J. Neuhaus, D. Bauer, J. Zhang, A. Killi, J. Kleinbauer, M. Kumkar, S. Weiler, M. Guina, D.H. Sutter, T. Dekorsy, Opt. Express 16, 20530 (2008). URL http://www.opticsexpress.org/ abstract.cfm?URI=0e-16-25-20530

31. J. Neuhaus, D. Bauer, J. Kleinbauer, A. Killi, D. Sutter, T. Dekorsy, J. Opt. Soc. Am. B, Opt. Phys. 27, 65 (2010) 